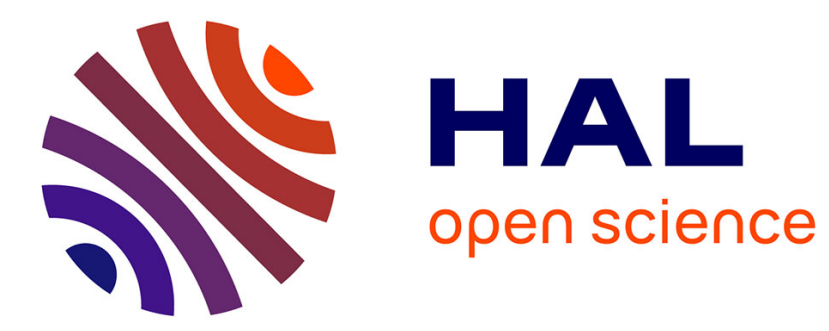

\title{
On the microscopic theory of phase coexistence
}

Salvador Miracle-Sole

\section{To cite this version:}

Salvador Miracle-Sole. On the microscopic theory of phase coexistence. XIII Sitges Conference, 1994, Sitges (Barcelona), Spain. pp.312-322. hal-00706955

\section{HAL Id: hal-00706955 https://hal.science/hal-00706955}

Submitted on 11 Jun 2012

HAL is a multi-disciplinary open access archive for the deposit and dissemination of scientific research documents, whether they are published or not. The documents may come from teaching and research institutions in France or abroad, or from public or private research centers.
L'archive ouverte pluridisciplinaire HAL, est destinée au dépôt et à la diffusion de documents scientifiques de niveau recherche, publiés ou non, émanant des établissements d'enseignement et de recherche français ou étrangers, des laboratoires publics ou privés. 


\title{
Centre de Physique Théorique ${ }^{1}$, CNRS Luminy, Case 907 \\ F-13288 Marseille - Cedex 9
}

\section{ON THE MICROSCOPIC THEORY OF PHASE COEXISTENCE}

\author{
Salvador MIRACLE-SOLE ${ }^{2}$
}

\begin{abstract}
Some rigorous results concerning the microscopic theory of interfaces and crystal shapes in classical lattice systems are reported.

Published in 25 Years of Non-Equilibrium Statistical Mechanics, J.J. Brey, J. Marro, J.M. Rubi, M. San Miguel (Eds.), Lecture Notes in Physics vol. 445, Springer, Berlin, 1995 (ISBN 3-540-59158-3), pp. 312-322. Proceedings of the XIII Sitges Conference, held in Sitges, Barcelona, Spain, 13-17 June 1994.

Keywords: Lattice systems; interfaces; surface tension; step free energy; equilibrium crystals.

October 1994

CPT-94/P.3077

anonymous ftp or gopher : cpt.univ-mrs.fr
\end{abstract}

\footnotetext{
${ }^{1}$ Unité Propre de Recherche 7061

${ }^{2}$ Centre de Physique Théorique, CNRS, Marseille
} 


\title{
ON THE MICROSCOPIC THEORY OF PHASE COEXISTENCE
}

\author{
Salvador Miracle SolÉ \\ Centre de Physique Théorique, CNRS Luminy, Case 907 \\ F-13288 Marseille Cedex 9, France
}

ABSTRACT: Some rigorous results concerning the microscopic theory of interfaces and crystal shapes in classical lattice systems are reported.

\section{INTRODUCTION}

It is known that the equilibrium shape of a crystal is obtained, according to the Gibbs thermodynamic theory, by minimizing the total surface free energy associated to the crystal-medium interface, and that this shape is given by the Wulff construction, provided one knows the anisotropic surface tension (or interfacial free energy per unit area). It is therefore important, even if a complete microscopic derivation of the Wulff construction within statistical mechanics has been proved only for some two-dimensional lattice models (see the recent work by Dobrushin et al. [1,2] and also Ref.3), to study the properties of the surface tension $\tau(\mathbf{n})$, as a function of the unit vector $\mathbf{n}$ which specifies the orientation of the interface with respect to the crystal axes. In the first approximation the crystal can be modelled by a lattice gas. In these notes, we shall present some new rigorous results on this subject which relate, in particular, to the problem of the appearance of plane facets in the Wulff equilibrium shape. For this purpose several aspects of the microscopic theory of interfaces are analysed, and another important quantity in this theory, the step free energy, is investigated (a complete version of this work will appear later [4]). In the last Section we shall report on some recent developments related to the theory of crystal growth.

\section{Gibbs States AND INTERFACES}

First we recall some classical results about the Gibbs states of the Ising model (to some extent these results were already discussed in Ref. 5). The 
model is defined on the cubic lattice $\mathcal{L}=\mathbb{Z}^{3}$, with configuration space $\Omega=\{-1,1\}^{\mathcal{L}}$. The value $\sigma(i)$ is the spin at the site $i$. The energy of a configuration $\sigma_{\Lambda}=\{\sigma(i), i \in \Lambda\}$, in a finite subset $\Lambda \subset \mathcal{L}$, under the boundary conditions $\bar{\sigma} \in \Omega$, is

$$
H_{\Lambda}\left(\sigma_{\Lambda} \mid \bar{\sigma}\right)=-\sum_{\langle i, j\rangle \cap \Lambda \neq \emptyset} \sigma(i) \sigma(j)
$$

where $\langle i, j\rangle$ are pairs of nearest neighbour sites and $\sigma(i)=\bar{\sigma}(i)$ if $i \notin \Lambda$. The partition function, at the inverse temperature $\beta=1 / k T$, is given by

$$
Z^{\bar{\sigma}}(\Lambda)=\sum_{\sigma_{\Lambda}} \exp \left(-\beta H_{\Lambda}\left(\sigma_{\Lambda} \mid \bar{\sigma}\right)\right)
$$

It is known that this model presents, at low temperatures $T<T_{c}$ (where $T_{c}$ is the critical temperature), two distinct thermodynamic pure phases, a positively and a negatively magnetized phase. This means two extremal translation invariant Gibbs states, which correspond to the limits, when $\Lambda \rightarrow$ $\infty$, of the finite volume Gibbs measures $Z^{\bar{\sigma}}(\Lambda)^{-1} \exp \left(-H_{\Lambda}\left(\sigma_{\Lambda} \mid \bar{\sigma}\right)\right)$, with boundary conditions $\bar{\sigma}$ equal to the ground configurations $(+)$ and $(-)$ (such that $\bar{\sigma}(i)=1$ and $\bar{\sigma}(i)=-1$ for all $i \in \mathcal{L})$, respectively. On the other side, if $T \geq T_{c}$, the Gibbs state is unique.

Each configuration inside $\Lambda$ can be geometrically described by specifying the Peierls contours, i. e., the boundaries between the spin 1 and spin -1 regions, which, under the above boundary conditions, are closed surfaces. The energy of the configuration is equal to twice the total area of the contours. The contours can be viewed as defects, or excitations, with respect to the ground states of the system, and are a basic tool for the investigation of the model at low temperatures.

In order to study the interface between the two pure phases one needs to construct a state describing the coexistence of these phases. Let $\Lambda$ be a parallelepiped of sides $L_{1}, L_{2}, L_{3}$, parallel to the axes, and centred at the origin of $\mathcal{L}$, and let $\mathbf{n}=\left(n_{1}, n_{2}, n_{3}\right)$ be a unit vector in $\mathbb{R}^{3}$, such that $n_{3} \neq 0$. Introduce the mixed boundary conditions $( \pm, \mathbf{n})$, for which $\bar{\sigma}(i)=1$ if $i \cdot \mathbf{n} \geq$ 0 , and $\bar{\sigma}(i)=-1$ if $i \cdot \mathbf{n}<0$. These boundary conditions force the system to produce a defect going transversely through the box $\Lambda$, a big Peierls contour that can be interpreted as a microscopic interface. The other defects that appear above and below can be described by closed contours inside the pure phases. 
Consider now the microscopic interface orthogonal to the direction $\mathbf{n}_{0}=$ $(0,0,1)$. At low temperatures $T>0$, we expect this interface, which at $T=0$ coincides with the plane $i_{3}=-1 / 2$, to be modified by deformations. It can be described by means of its defects, or excitations, with respect to the interface at $T=0$. These defects, called walls, form the boundaries (which may have some width), between the smooth plane portions of the interface. In this way the interface structure, with its probability distribution in the corresponding Gibbs state, may then be interpreted as a "gas of walls" on a two-dimensional lattice.

Using the Peierls method, Dobrushin [6] proved the dilute character of this gas at low temperatures, which means that the interface is essentially flat (or rigid). The considered boundary conditions yield indeed a non translation invariant Gibbs state. Furthermore, cluster expansion techniques have been applied by Bricmont et al. [7,8], to study the interface structure in this case (see also Ref. 9).

The same analysis applied to the two-dimensional model shows a different behaviour at low temperatures. In this case the walls belong to a one-dimensional lattice, and Gallavotti [10] proved that the microscopic interface undergoes large fluctuations of order $\sqrt{L_{1}}$. The interface does not survive in the thermodynamic limit, $\Lambda \rightarrow \infty$, and the corresponding Gibbs state is translation invariant. Moreover, the interface structure can be studied by means of a cluster expansion for any orientation of the interface (see also Ref. 11). Such a problem in the three-dimensional case leads to very difficult problems of random surfaces. This is one of the serious difficulties which face the attempts to generalise the work by Dobrushin et al. [1] to the three-dimensional Ising model, since a very accurate description of the microscopic interface for any orientation $\mathbf{n}$ is needed in this work.

\section{The SURFACE TENSION}

The free energy, per unit area, due to the presence of the interface, is the surface tension. It can be defined by

$$
\tau(\mathbf{n})=\lim _{L_{1}, L_{2} \rightarrow \infty} \lim _{L_{3} \rightarrow \infty}-\frac{n_{1}}{\beta L_{1} L_{2}} \ln \frac{Z^{( \pm, \mathbf{n})}(\Lambda)}{Z^{(+)}(\Lambda)}
$$

Notice that in this expression the volume contributions proportional to the free energy of the coexisting phases, as well as the boundary effects, cancel, and only the contributions to the free energy of the interface are left. 
TheOREm 1. The thermodynamic limit $\tau(\mathbf{n})$, of the interfacial free energy per unit area, exists, and, as a function of $\mathbf{n}$, extends by positive homogeneity to a convex function $f(\mathbf{x})=|\mathbf{x}| \tau(\mathbf{x} /|\mathbf{x}|)$ defined for any vector $\mathbf{x} \in \mathbb{R}^{3}$.

A proof of these statements was given in Ref. 12 using correlation inequalities (this being the reason for their general validity). Moreover, we know (from Refs. 13 and 14 and the convexity condition) that $\tau(\mathbf{n})$ is strictly positive for $T<T_{c}$ and that it vanishes if $T \geq T_{c}$.

The convexity of $f(\mathbf{x})$ may be interpreted as a thermodynamic stability condition. It is equivalent (as shown in Ref.12) to the pyramidal inequality for the function $\tau(\mathbf{n})$. This condition, introduced in Ref. 1 for the twodimensional Ising model (triangular inequality), was conjectured to hold true in general situations in Ref. 15.

According to the Wulff construction, the equilibrium shape of a crystal is given by

$$
\mathcal{W}=\left\{\mathbf{x} \in \mathbb{R}^{3} \mid \mathbf{x} \cdot \mathbf{n} \leq \tau(\mathbf{n}) \text { for every } \mathbf{n}\right\}
$$

where $\tau(\mathbf{n})$ is the surface tension of the interface orthogonal to $\mathbf{n}$. One obtains in this way the shape which has the minimum surface free energy for a given volume. Defined as the intersection of closed half-spaces, $\mathcal{W}$ is a closed bounded convex set, i.e., a convex body.

It turns out that if $f(\mathbf{x})$ is a convex function, then it is also the Minkowski's support function of the convex body $\mathcal{W}$ (i. e., $f(\mathbf{x})=\sup _{\mathbf{y} \in \mathcal{W}} \mathbf{x} \cdot \mathbf{y}$ ). As a consequence of this fact the following macroscopic properties can be proved.

Theorem 2. Assume that the convexity condition is satisfied. A facet orthogonal to the direction $\mathbf{n}_{0}$ appears in the Wulff equilibrium crystal shape if, and only if, the derivative $\partial \tau(\theta, \phi) / \partial \theta$ is discontinuous at the point $\theta=0$, for all $\phi$. Moreover, the one-sided derivatives $\partial \tau(\theta, \phi) / \partial \theta$, at $\theta=0^{+}$and $\theta=0^{-}$, exist, and determine the shape of the facet.

Here, the function $\tau(\mathbf{n})=\tau(\theta, \phi)$ is expressed in terms of the spherical co-ordinates $0 \leq \theta \leq \pi, 0 \leq \phi \leq 2 \pi$ of $\mathbf{n}$, the vector $\mathbf{n}_{0}$ being taken as the polar axis. Actually, the shape of the facet is given by

$$
\mathcal{F}=\left\{\mathbf{x} \in \mathbb{R}^{2} \mid \mathbf{x} \cdot \mathbf{m} \leq \mu(\mathbf{m}) \text { for every } \mathbf{m}\right\}
$$

where $\mathbf{m}=(\cos \phi, \sin \phi) \in \mathbb{R}^{2}$ and $\mu(\phi)=(\partial / \partial \theta)_{\theta=0^{+}} \tau(\theta, \phi)$. 


\section{THE STEP FREE ENERGY}

The step free energy plays, also, an important role in the problem under consideration. It is defined, again using appropriate boundary conditions, as the free energy associated with the introduction of a step of height 1 on the interface. This quantity can be regarded as an order parameter for the roughening transition, analogous, in some sense, to the surface tension in the case of a phase transition.

Let us consider again the interface orthogonal to a lattice axis, which, as we know from Section 2, is rigid at low temperatures. It is believed, that at higher temperatures, but before reaching the critical temperature $T_{c}$, the fluctuations of the considered interface become unbounded when the volume tends to infinity, so that the corresponding Gibbs state in the thermodynamic limit is translation invariant. The interface undergoes a roughening phase transition at a temperature $T=T_{R}$.

Approximate methods, used by Weeks et al. [16], suggest $T_{R} \sim 0.53 T_{c}$, a temperature slightly higher then $T_{c}^{d=2}$ (the critical temperature of the twodimensional Ising model), and actually van Beijeren [17] proved, using correlation inequalities, that $T_{R} \geq T_{c}^{d=2}$. The analogous result for the step free energy, i. e., that $\tau^{\text {step }}>0$ if $T<T_{c}^{d=2}$, was proved in Ref. 18, as well as that $\tau^{\text {step }}=0$ if $T \geq T_{c}$. Since then, however, it appears to be no proof of the fact that $T_{R}<T_{c}$.

At present one is able to study rigorously the roughening transition only for some simplified models of the microscopic interface. Thus, Fröhlich and Spencer [19] have proved this transition for the SOS (solid-on-solid) model, and several restricted SOS models, which are exactly solvable, have also been studied in this context (these models are reviewed in Refs. 20 and 21).

In order to define the step free energy we consider the box $\Lambda$ as above and and introduce the (step, $\mathbf{m})$ boundary conditions, associated to the unit vectors $\mathbf{m}=(\cos \phi, \sin \phi) \in \mathbb{R}^{2}$, by

$$
\bar{\sigma}(i)= \begin{cases}1 & \text { if } i>0 \text { or if } i_{3}=0 \text { and } i_{1} m_{1}+i_{2} m_{2} \geq 0 \\ -1 & \text { otherwise }\end{cases}
$$

Then, the step free energy, for a step orthogonal to $\mathbf{m}$ ( $\operatorname{such}$ that $m_{2} \neq 0$ ), is

$$
\tau^{\text {step }}(\phi)=\lim _{L_{1} \rightarrow \infty} \lim _{L_{2} \rightarrow \infty} \lim _{L_{3} \rightarrow \infty}-\frac{\cos \phi}{\beta L_{1}} \ln \frac{Z^{(\text {step }, \mathbf{m})}(\Lambda)}{Z^{\left( \pm, \mathbf{n}_{0}\right)}(\Lambda)}
$$


Clearly, this expression represents the residual free energy due to the considered step, per unit length.

When considering the configurations under the $($ step, $\mathbf{m})$ boundary conditions, the step may be viewed as a defect on the rigid interface. It is in fact, a long wall going from one side to the other of the box $\Lambda$. A more careful description of it can be obtained as follows. At $T=0$, the step parallel to the axis (i. e., for $\mathbf{m}=(0,1)$ ) is a perfectly straight step of height 1 . At a low temperature $T>0$, some deformations appear, connected by straight portions of height 1 . The step structure, with its probability distribution in the corresponding Gibbs state, can then be described as a "gas" of these defects (to be called step-jumps), on a one-dimensional lattice. This description, somehow similar to the description of the interface of the two-dimensional Ising model used by Gallavotti [10], is valid, in fact, for any orientation $\mathbf{m}$ of the step. It can be shown that the gas of step-jumps is a dilute gas at low temperature and, as a consequence of this fact, cluster expansion techniques can be applied in order to study the step structure. Actually, the step-jumps are not independent since the rest of the system produces an effective interaction between them. Nevertheless, this interaction can be treated by applying the low temperature expansion, in terms of walls, for the rigid interface, to the regions of the interface lying at both sides of the step. From this analysis one gets the following result.

THEOREM 3. If the temperature is low enough (i.e., if $T \leq T_{0}$ where $T_{0}>0$ is a given constant), then the step free energy $\tau^{\text {step }}$, exists in the thermodynamic limit, and extends by positive homogeneity to a strictly convex function. Moreover, it can be expressed in terms of an analytic function of $T$, for which a convergent power series expansion can be obtained from the above mentioned cluster expansion.

In fact,

$$
\begin{aligned}
\tau^{\text {step }}(\mathbf{m})= & 2 J\left(\left|m_{1}\right|+\left|m_{2}\right|\right)-(1 / \beta)\left(\left(\left|m_{1}\right|+\left|m_{2}\right|\right) \ln \left(\left|m_{1}\right|+\left|m_{2}\right|\right)\right. \\
& \left.-\left|m_{1}\right| \ln \left|m_{1}\right|-\left|m_{2}\right| \ln \left|m_{2}\right|\right)-(1 / \beta) \varphi_{\mathbf{m}}(\beta)
\end{aligned}
$$

where $\varphi_{\mathbf{m}}$ is an analytic function of $z=e^{-2 \beta}$, for $|z| \leq e^{-2 \beta_{0}}$. The first two terms in this expression, which represent the main contributions for $T \rightarrow 0$, come from the ground state of the system under the considered boundary conditions. The first term can be recognised as the residual energy of the 
step at zero temperature and, the second term, as $-(1 / \beta)$ times the entropy of this ground state. The same two terms occur in the surface tension of the two-dimensional Ising model (see Ref. 22). By considering the lowest energy excitations, it can be seen that $\varphi_{\mathbf{m}}$ is $O\left(e^{-4 \beta}\right)$, and also, that the first term in which this series differs from the series associated to the surface tension of the two-dimensional Ising model, is $O\left(e^{-12 \beta}\right)$.

\section{FACETS in the EQUilibrium CRYSTAL}

The roughness of an interface should be apparent when considering the shape of the equilibrium crystal associated with the system. One knows that a typical equilibrium crystal at low temperatures has smooth plane facets linked by rounded edges and corners. The area of a particular facet decreases as the temperature is raised and the facet finally disappears at a temperature characteristic of its orientation. The reader will find information and references on equilibrium crystals in the review articles of Refs. 20, 21, 23 and 24.

It can be argued, as discussed below, that the roughening transition corresponds to the disappearance of the facet whose orientation is the same as that of the considered interface. The exactly solvable SOS models mentioned above, for which the function $\tau(\mathbf{n})$ has been computed, are interesting examples of this behaviour (this subject has been reviewed in Ref. 12, Chapter VII). For the three-dimensional Ising model, Bricmont et al. [25] have proved a correlation inequality which establish $\tau^{\text {step }}$ as a lower bound to the one-sided derivative $\partial \tau(\theta) /\left.\partial \theta\right|_{\theta=0^{+}}\left(\right.$here $\tau^{\text {step }}=\tau^{\text {step }}(0,1)$ and $\tau(\theta)=\tau(0, \sin \theta, \cos \theta)$ ). Thus $\tau^{\text {step }}>0$ implies a kink in $\tau(\theta)$ at $\theta=0$ and, according to the Wulff construction, a facet is expected.

In fact, $\tau^{\text {step }}$ should be equal to this derivative. This is reasonable, since the increment in surface tension of an interface tilted by an angle $\theta$, with respect to the surface tension of the rigid interface, can be approximately identified, for $\theta$ small, with the free energy of a "gas of steps" (the density of the steps being proportional to $\theta$ ). And, again, if the interaction between the steps can be neglected, the free energy of this gas can be approximated by the sum of the individual free energies of the steps.

As a result of the methods described in Section 3, it is possible to study the statistical mechanics of this "gas of steps", and to derive the following result. 
Theorem 4. For $T<T_{0}$, we have

$$
\partial \tau(\theta, \phi) /\left.\partial \theta\right|_{\theta=0^{+}}=\tau^{\text {step }}(\phi)
$$

i. e., the step free energy equals the one-sided angular derivative of the surface tension.

It is natural to expect that this equality is true for any $T$ less than $T_{R}$, and that for $T \geq T_{R}$, both sides in the equality vanish, and thus, the disappearance of the facet is involved (these facts can be proved for certain SOS models of interfaces using correlation inequalities [26]). However, the condition that the temperature is low enough is important here. Only when it is fulfilled we have the full control on the equilibrium probabilities that is needed in the proofs.

The above relation, together with the discussion in Section 3, implies that one obtains the shape of the facet by means of the two-dimensional Wulff construction applied to the step free energy $\tau^{\text {step }}(\mathbf{m})$. Namely,

$$
\mathcal{F}=\left\{\mathbf{x} \in \mathbb{R}^{2} \mid \mathbf{x} \cdot \mathbf{m} \leq \tau^{\text {step }}(\mathbf{m}) \text { for every } \mathbf{m}\right\}
$$

Then, from the properties of $\tau^{\text {step }}$, it follows that the facet has a smooth boundary without straight segments and, therefore, that the crystal shape presents rounded edges and corners.

\section{NUCLEATION AND GROWING CRYSTALS}

The phenomenon of nucleation takes place when a thermodynamic system, instead of undergoing a phase transition, stays in a metastable phase. The stable phase emerges via the formation of a suitable, sufficiently large droplet (nucleus). In fact, due to the competition, already at a microscopic level, between the volume free energy and the surface tension, small droplets have a tendency to shrink whereas large ones prefer to grow. The activation energy necessary for the formation of a critical nucleus, and the time which takes to overcome this energy barrier, become larger and larger, together with the size of the nucleus, as the parameters tend to their values at the coexistence point. This explains the very long life of a metastable state.

Different aspects of this important subject have recently been discussed from a rigorous point of view. We present here a brief account of the work by Kotecký and Olivieri [27] concerning the droplet dynamics in the Ising 
model (see also Ref. 28, by the same authors, where a more complex case is discussed). The subject was previously developped by Neves and Schonmann $[29,30]$, following the approach introduced by Cassandro et al [31].

The anisotropic two-dimensional Ising model, with vertical and horizontal coupling constants $J_{1}>J_{2}>0$, in the presence of a very small magnetic field $h>0$, is studied. Let $\Lambda$ be a square box of side $L$, with periodic boundary conditions, and let $H(\sigma)$ denote the energy of a configuration $\sigma \in \Omega_{\Lambda}=$ $\{-1,1\}^{\Lambda}$. We suppose that the volume is sufficiently large, $L>\left(2 J_{1} / h\right)^{3}$.

A discrete time stochastic dynamics is then considered for this model. Namely, the Metropolis dynamics defined by the following updating rule: Given a configuration $\sigma$ at time $t$ one first chooses randomly a site $i \in \Lambda$ with uniform probability $1 /|\Lambda|$. Then one flips the spin at site $i$ with probability

$$
\exp \left(-\beta \max \left\{H\left(\sigma^{(i)}\right)-H(\sigma), 0\right\}\right)
$$

where $\sigma^{(i)}(j)=\sigma(j)$, whenever $j \neq i$, and $\sigma^{(i)}(j)=-\sigma(j)$, for $j=i$. This dynamics is reversible with respect to the Gibbs measure.

The nucleation from a metastable state is studied for this model in the limit of very low temperatures ( $h$ fixed). It turns out that the critical nucleus, as well as the configurations on a typical path to it, differ from the Wulff shape of an equilibrium droplet. The critical droplet is in fact a square of side $\ell^{*}=\left[2 J_{2} / h\right]+1$ ([.] denotes the integer part), while the Wulff shape is a rectangle of sides proportional to $J_{1}, J_{2}$ (agreement could be expected, however, in the more customary region $T$ fixed, small, and $h \rightarrow 0$ ).

A path of the process is a sequence $\omega=\sigma_{0}, \sigma_{1}, \ldots, \sigma_{t}, \ldots$ of configurations in $\Omega_{\Lambda}$. We suppose that the process starts at the configuration $\sigma_{0}=(-)$ (all spins $\sigma_{0}(i)$ in $\Lambda$ equal to -1$)$. We are interested in the first passage from the configuration $(-)$ to the configuration $(+)$, which takes place between the moments $\tau_{(-)}=\max \left\{t<\tau_{(+)} \mid \sigma_{t}=(-)\right\}$ and $\tau_{(+)}=\min \left\{t \mid \sigma_{t}=(+)\right\}$.

The configurations $r\left(\ell_{1}, \ell_{2}\right)$, which have a rectangle of sides $\ell_{1}, \ell_{2}$ as unique Peierls contour, play a particular role in the process. They correspond to the local minima of the energy (in the sense that one spin flip increases the energy). Now, the probability that starting from a given local minimum $Q$ the system goes to a neighbouring local minimum $Q^{\prime}$, is determined by the energy barrier $H(S)-H(Q)$, where $S$ is any configuration at which the energy on a path from $Q$ to $Q^{\prime}$ reaches its maximum, but with the path chosen to minimalise it. In other words, the configurations in $S$ are the local 
saddle points for which the minimax

$$
\min _{\omega: Q \rightarrow Q^{\prime}} \max _{\sigma \in \omega} H(\sigma)
$$

is attained (here $\omega: Q \rightarrow Q^{\prime}$ denotes a generic path with successive spin flips starting from a configuration in $Q$ and ending at $\left.Q^{\prime}\right)$. The considered probability is proportional to $\exp [-\beta(H(S)-H(Q))]$. On the other side, the system in the local minimum $Q$ is likely to "stay" in its basin of attraction for a time of order $\exp [\beta(H(\bar{S})-H(Q))]$, where $\bar{S}$ is the local saddle point with lowest energy through which it can escape from the local minimum $Q$, not necessarily in "the direction" of $Q^{\prime}$. These are the basic mechanisms which determine the local dynamics. The task is then to find the class of paths which describe the most probable evolution.

Let us consider the probability of reaching a global saddle point, defined by the same minimax condition extended to all paths from the configuration $(-)$ to the configuration $(+)$. These configurations give rise to the critical nucleus.

It can be seen that the set of all global saddle points coincides with the set $\mathcal{P}$ of all configurations having as unique contour a rectangle, of sides $\ell^{*}, \ell^{*}-1$, or $\ell^{*}-1, \ell^{*}$, with a unit square attached to one of its longer sides. The relative energy of any $\bar{\sigma} \in \mathcal{P}$ is

$$
\Gamma=H(\bar{\sigma})-H((-))=2\left(J_{1}+J_{2}\right) \ell^{*}-h\left(\left(\ell^{*}\right)^{2}-\ell^{*}+1\right)
$$

It is proved that the first excursion from $(-)$ to $(+)$ passes through a configuration from $\mathcal{P}$ and the time needed for this to happen is of the order $\exp (\beta \Gamma)$. Introducing the time $\tau_{\mathcal{P}}=\min \left\{t>\tau_{(-)} \mid \sigma_{t} \in \mathcal{P}\right\}$, the precise statement can be formulated as follows.

TheOREM 5. We have

$$
\lim _{T \rightarrow 0} \operatorname{Prob}\left[\tau_{\mathcal{P}}<\tau_{(+)}\right]=1
$$

and, moreover, for any $\epsilon>0$,

$$
\lim _{T \rightarrow 0} \operatorname{Prob}\left[\exp (\beta(\Gamma-\epsilon))<\tau_{\mathcal{P}}<\exp (\beta(\Gamma+\epsilon))\right]=1
$$

In addition, from the arguments in the proof of this result, one is getting very detailed information about a typical path followed by the process $\sigma_{t}$ during its first excursion from $(-)$ to $(+)$. 
a) First it passes through a monotonously growing sequence of subcritical rectangles $r\left(\ell_{1}, \ell_{2}\right)$, such that $\left|\ell_{1}-\ell_{2}\right|=0$ or 1 , up to the critical square $r\left(\ell^{*}, \ell^{*}\right)$.

b) After the vertical edge stays constant at the value $\ell^{*}$ while the horizontal edge grows up to L. Finally the vertical edge grows from $\ell^{*}$ to $L$.

The precise statements involve the notion of $\epsilon$-typical path, that is determined not only in terms of geometrical properties, but also with specified times of passage (by means of bounds analogous to those used above for $\tau_{\mathcal{P}}$ ) through certain configurations. The path is an $\epsilon$-typical path (for any given $\epsilon>0$ ) with a probability which tends to 1 when $T \rightarrow 0$.

Finally, let us mention that Schonmann [32] has recently discussed the regime in which the temperature is kept fixed and the field $h>0$ is scaled to zero. As conjectured in Ref. 33, for the Ising model in any dimension $d \geq 2$, if the temperature is low enough, the relaxation time goes in this regime as an exponential of $1 / h^{d-1}$. Moreover, before a time which grows also as an exponential of $1 / h^{d-1}$ the system stays in a metastable situation.

ACKNOWLEDGements: It is a pleasure to thank Roman Kotecký for very valuable discussions.

\section{REFERENCES}

1. R.L. Dobrushin, R. Kotecký and S.B. Shlosman: The Wulff construction: a global shape from local interactions. Providence: Amer. Math. Soc. 1992.

2. R.L. Dobrushin, R. Kotecký and S.B. Shlosman: J. Stat. Phys. 72, 1 (1993)

3. C.E. Pfister: Helv. Phys. Acta 64, 953 (1991)

4. S. Miracle-Sole: Surface tension, step free energy and facets in the equilibrium crystal. Marseille: Preprint, 1994.

5. S. Miracle-Sole: In: Critical phenomena, Sitges international school. J. Brey and R.B. Jones eds. Berlin: Springer, 1976.

6. R.L. Dobrushin: Theory Prob. Appl. 17, 582 (1972)

7. J. Bricmont, J.L. Lebowitz, C.E. Pfister and E. Olivieri: Commun. Math. Phys. 66, 1 (1979).

8. J. Bricmont, J.L. Lebowitz and C.E. Pfister: Commun. Math. Phys. 66, 21 (1979); Commun. Math. Phys. 69, 267 (1979) 
9. P. Holický, R. Kotecký and M. Zahradník: J. Stat. Phys. 50, 755 (1988)

10. G. Gallavotti: Commun. Math. Phys. 27, 103 (1972)

11. J. Bricmont, J.L. Lebowitz and C.E. Pfister: J. Stat. Phys. 26, 313 (1981)

12. A. Messager, S. Miracle-Sole and J. Ruiz: J. Stat. Phys. 67, 449-470 (1992)

13. J. Bricmont, J.L. Lebowitz and C.E. Pfister: Ann. Acad. Sci. New York 337, 214 (1980)

14. J.L. Lebowitz and C.E. Pfister: Phys. Rev. Lett. 46, 1031 (1981)

15. R.L. Dobrushin and S.B. Shlosman: In: Ideas and methods in mathematical analysis, stochastics and applications. S. Albeverio, S.E. Fenstad, H. Holden and T. Lindstrom eds. Cambridge: University Press, 1991

16. J.D. Weeks, G.H. Gilmer and H.J. Leamy: Phys. Rev. Lett. 31, 549 (1973)

17. H. van Beijeren: Commun. Math. Phys. 40, 1 (1975)

18. J. Bricmont, J.R. Fontaine and J.L. Lebowitz: J. Stat. Phys. 29, 193 (1982)

19. J. Fröhlich and T. Spencer: Commun. Math. Phys. 81, 527 (1981)

20. H. van Beijeren and I. Nolden: In: Topics in current Physics, Vol. 43. W. Schommers and P. von Blackenhagen eds. Berlin: Springer 1987.

21. D.B. Abraham: In: Phase transitions and critical phenomena, Vol. 10. C. Domb and J.L. Lebowitz eds. London: Academic Press 1986.

22. J.E. Avron, H. van Beijeren, L.S. Shulman and R.K.P. Zia: J. Phys. A: Mat. Gen. 15, L81 (1982)

23. C. Rottman and M. Wortis: Phys. Rep. 103, 59 (1984)

24. R. Kotecký: In: IX international congress of mathematical Physics. B. Simon A. Truman and I.M. Davies eds. Bristol: Adam Hilger 1889.

25. J. Bricmont, A. El Mellouki and J. Fröhlich: J. Stat. Phys. 42, 743 (1986)

26. S. Miracle-Sole: In preparation

27. R. Kotecký and E. Olivieri: J. Stat. Phys. 70, 1121 (1993).

28. R. Kotecký and E. Olivieri: J. Stat. Phys. 75, 409 (1994)

29. E.J. Neves and R.H. Schonmann: Commun. Math. Phys. 137, 209 (1991)

30. R.H. Schonmann: Commun. Math. Phys. 147, 231 (1991)

31. M. Cassandro, A. Galves, E. Olivieri and M.E. Vares: J. Stat. Phys. 35, $603(1984)$ 
32. R.H. Schonmann: In: Probability and phase transitions. G. Grimmet, ed. Dordrecht: Kluwer Academic Publishers, 1994.

33. M. Aizenman and J.L. Lebowitz: J. Phys. A: Math. Gen. 21, 3801 (1988) 